\title{
Threshold Resummation Effects in the Polarized Drell-Yan Mechanism
}

\author{
Hiroshi Yokoya and Werner Vogelsang
}

Presented at SPIN 2006

Kyoto, Japan

October 2-7, 2006

\author{
Physics Department \\ Nuclear Theory Group \\ Brookhaven National Laboratory \\ P.O. Box 5000 \\ Upton, NY 11973-5000 \\ www.bnl.gov
}

\footnotetext{
Notice: This manuscript has been authored by employees of Brookhaven Science Associates, LLC under Contract No. DE-AC0298CH10886 with the U.S. Department of Energy. The publisher by accepting the manuscript for publication acknowledges that the United States Government retains a non-exclusive, paid-up, irrevocable, world-wide license to publish or reproduce the published form of this manuscript, or allow others to do so, for United States Government.purposes.
} 


\section{DISCLAIMER}

This report was prepared as an account of work sponsored by an agency of the United States Government. Neither the United States Government nor any agency thereof, nor any of their employees, nor any of their contractors, subcontractors, or their employees, makes any warranty, express or implied, or assumes any legal liability or responsibility for the accuracy, completeness, or any third party's use or the results of such use of any information, apparatus, product, or process disclosed, or represents that its use would not infringe privately owned rights. Reference herein to any specific commercial product, process, or service by trade name, trademark, manufacturer, or otherwise, does not necessarily constitute or imply its endorsement, recommendation, or favoring by the United States Governiment or any agency thereof or its contractors or subcontractors. The views and opinions of authors expressed herein do not necessarily state or reflect those of the United States Government or any agency thereof. 


\title{
Threshold Resummation Effects in the Polarized Drell-Yan Mechanism
}

\author{
Hiroshi Yokoya ${ }^{*}$ and Werner Vogelsang ${ }^{\dagger}$ \\ ${ }^{*}$ Dept. of Physics, Niigata University, Niigata 950-2181, Japan \\ ${ }^{\dagger}$ Physics Department, Brookhaven National Laboratory, Upton, New York 11973, U.S.A.
}

\begin{abstract}
We present theoretical predictions for the cross sections and spin asymmetries in dilepton pair production in transversely polarized $p p$ and $\bar{p} p$ collisions. We use the available fixed-order corrections as well as the all-order resummation of threshold logarithms for the pair mass and rapidity distributions. Numerical results for $p p$ collisions at $\sqrt{s}=10 \mathrm{GeV}$ at J-PARC and for $\bar{p} p$ collisions at $\sqrt{s}=14.5 \mathrm{GeV}$ at GSI-PAX are given.
\end{abstract}

\section{INTRODUCTION}

Recently, new experiments in polarized hadron collisions have been proposed for GSI [1] $(\vec{p} p)$ and J-PARC [2] $(p p)$. The experiments would be at relatively modest collision energies, $\sqrt{S}=14.5 \mathrm{GeV}$ at GSI-PAX and $\sqrt{S}=10 \mathrm{GeV}$ at J-PARC. One of the main purposes of these experiments is the measurement of transverse spin asymmetries in the Drell-Yan process; in order to get information on the transversely polarized parton distribution functions (PDFs) of the nucleon. At these energies, perturbativeQCD (pQCD) corrections as well as power-suppressed contributions may be important and require careful theoretical study.

In this report, we present recent studies of pQCD corrections to the invariant-mass and rapidity distributions of Drell-Yan cross sections and transverse double-spin asymmetries. In particular, we consider the all-order resummation of large "threshold" logarithms [3], to next-to-next-to-leading logarithmic (NNLL) accuracy. For further details about some of the results presented below, including a discussion of possible nonperturbative effects to the cross section, see [4].

\section{THRESHOLD RESUMMATION}

The invariant-mass distribution of Drell-Yan lepton pairs can be written in terms of the PDFs and partonic hard-scattering cross sections as

$$
\frac{d \sigma}{d M^{2}}=N \sum_{a b} \int_{\tau}^{1} \frac{d x_{1}}{x_{1}} f_{a}\left(x_{1}, \mu\right) \int_{\tau / x_{1}}^{1} \frac{d x_{2}}{x_{2}} f_{b}\left(x_{2}, \mu\right) \omega_{a b}\left(z, \alpha_{s}(\mu), r\right) .
$$

The transversely polarized cross section, $\delta \sigma$ is written in an analogous manner. In (1), $\tau=M^{2} / S, z=\tau / x_{1} x_{2}$ and $r=M^{2} / \mu^{2}$, with $\mu$ the renormalization/factorization scale. $N$ is defined so that the $\mathscr{O}\left(\alpha_{s}^{0}\right)$ term becomes $\omega_{q \bar{q}}^{(0)}=\delta(1-z)$. The higher-order functions 
$\omega_{a b}^{(i)}$ have been calculated to $\mathscr{O}\left(\alpha_{s}^{2}\right)$ for the unpolarized cross section [5], and to $\mathscr{O}\left(\alpha_{s}^{1}\right)$ for the transversely polarized one [6].

The numerical size of the NNLO corrections for GSI or J-PARC kinematics amounts to more than three times the LO cross section at high $M$ [4]. It is known that these large corrections come from the threshold region where the partonic energy is just enough to produce the lepton pair of invariant mass $M$. In this region, large "threshold" logarithms arise. The systematic way of taking into account these logarithms to all orders, called "threshold resummation", has been developed in [3]. The resummation is achieved in Mellin-moment space, where it gives rise to a Sudakov exponent. Presently, the exponent for the Drell-Yan process is known to NNLL accuracy [7]. Defining $\omega_{a b}(n)=\int_{0}^{1} d z z^{n-1} \omega_{a b}(z)$, one has:

$$
\omega_{q \tilde{q}}^{\mathrm{res}}\left(n, \alpha_{s}, r\right)=C_{D Y}\left(\alpha_{s}, r\right) \exp \left[\frac{1}{\alpha_{s}} h_{q}^{(1)}(\lambda)+h_{q}^{(2)}(\lambda, r)+\alpha_{s} h_{q}^{(3)}(\lambda, r)\right],
$$

where $\lambda=b_{0} \alpha_{s} \ln n$. The detailed expressions for the $n$-independent coefficient $C_{D Y}$ and the functions $h_{q}^{(i)}$ may be found, e.g., in Ref. [7]. We note that $C_{D Y}$ is also known to exponentiate [8]. We use the "Minimal Prescription" [9] for dealing with the Landau pole at $\lambda=1 / 2$ in the resummed expression.

It is known that the resummation formula can be improved to include collinear (nonsoft) gluon effects $[10,11]$. In NLO, these correspond to terms $\propto \alpha_{s} \ln (n) / n$. They may be taken into account in the resummation by including certain subleading terms in the exponent, associated with DGLAP evolution of parton distributions. Through singlet mixing in evolution, these subleading terms also feed into the $q g$-subprocess [11]. We found that these effects are significant, especially for the case of $p p$ collisions at J-PARC.

Fig. 1(left) shows the expansions of the NNLL-resummed $K$-factor to fixed perturbative orders. We stress that the second- and third-order expansions are in good agreement with the full NLO and NNLO results, when the mixing is implemented. This shows that the higher-order corrections are indeed dominated by the threshold logarithms, and that the resummation is accurately reproducing the latter.
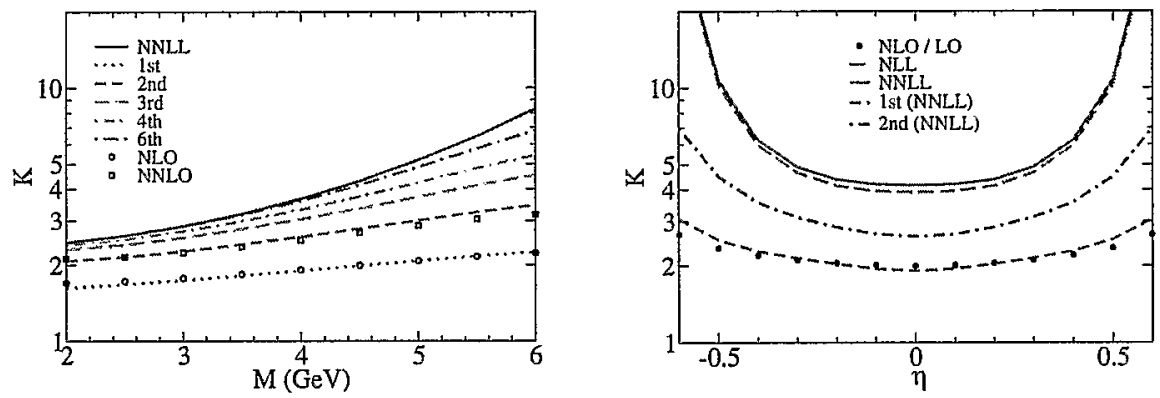

FIGURE 1. $K$-factors for the resummed cross section and its perturbative expansions for $p p$ collisions at $\sqrt{S}=10 \mathrm{GeV}$. Left: invariant-mass distribution, right: rapidity distribution at $M=5 \mathrm{GeV}$. The NLO (NNLO) $K$-factors are also plotted as circle (square) symbols. 


\section{RAPIDITY DISTRIBUTION}

We now consider the cross section differential in the lepton pair's rapidity,

$$
\frac{d \sigma}{d M^{2} d \eta}=N \sum_{a b} \int_{x_{1}^{0}}^{1} d x_{1} f_{a}\left(x_{1}, \mu\right) \int_{x_{2}^{0}}^{1} d x_{2} f_{b}\left(x_{2}, \mu\right) D_{a b}\left(x_{1}^{0}, x_{2}^{0}, x_{1}, x_{2}, \alpha_{s}\right)
$$

where $x_{1,2}^{0}=\sqrt{\tau} \mathrm{e}^{ \pm \eta}$. The $D_{a b}$ have been calculated perturbatively to $\mathscr{O}\left(\alpha_{s}^{2}\right)$ for the unpolarized cross section [12], and to $\mathscr{O}\left(\alpha_{s}\right)$ for the transversely polarized case [13]. The $\mathscr{O}\left(\alpha_{s}^{0}\right)$ term is simply $D_{q \bar{q}}^{(0)}=\delta\left(x_{1}-x_{1}^{0}\right) \delta\left(x_{2}-x_{2}^{0}\right)$. The application of the threshold resummation technique to rapidity distributions has been discussed in Ref. [14]. In addition to the usual Mellin transform in $\tau$, it makes use of a Fourier transform in $\eta$. The cross section in double-transform space can be written as

$$
\begin{aligned}
\tilde{\sigma}\left(n, m, \alpha_{s}, r\right) & \equiv \int_{0}^{1} d \tau \tau^{n-1} \int d \eta e^{i m \eta} \frac{d \sigma}{d M^{2} d \eta} \\
& =N \sum_{a b} f_{a}\left(n+\frac{i}{2} m, \mu\right) f_{b}\left(n-\frac{i}{2} m, \mu\right) \tilde{D}_{a b}\left(n, m, \alpha_{s}, r\right) .
\end{aligned}
$$

In the threshold limit, $\tilde{D}_{q \bar{q}}$ can be written in terms of the higher-order function $\omega_{q q \bar{q}}\left(n, \alpha_{s}\right)$ for the invariant-mass distribution discussed above. The resummation may then be performed as before. Details will be presented elsewhere. In Fig. 1 (right), we show the $K$-factor for the Drell-Yan rapidity distribution at NLO and resummed, for the case of $p p$ collisions at J-PARC. The $K$-factors increase toward larger $\eta$, since one approaches the threshold regime more closely there.

\section{TRANSVERSE DOUBLE SPIN ASYMMETRY}

The transverse double-spin asymmetry, $A_{T T}$ is defined as the ratio of the transversely polarized and unpolarized cross sections, differential with respect to the azimuthal angle of one lepton. Following Ref. [13], we model the transversity densities by saturating the Soffer inequality [15], using the GRV [16] unpolarized and GRSV [17] longitudinally polarized PDFs. For the GSI $\bar{p} p$ case, the higher-order QCD corrections cancel out to a large degree in $A_{T T}$, because the $q \vec{q}$-subprocess describes "valence-valence" scattering and hence dominates both the unpolarized and the transversely polarized cross section [4]. In Fig. 2, we show the corresponding $A_{T T}$ for $p p$ collisions at J-PARC. We found that in this case the asymmetry is less stable under higher-order corrections, because the $q \vec{q}$ scattering now involves sea quark distributions and is less dominant. The $q g$-subprocess contributes significantly to the unpolarized cross section, while it is absent in the transversely polarized one. Thus, a careful treatment of the corrections will be needed in order to extract the transversity densities from future $A_{T T}$ measurements at J-PARC. Also, a good understanding of the large- $x$ behavior of the gluon and sea-quark densities will be important. Details will be presented elsewhere. 

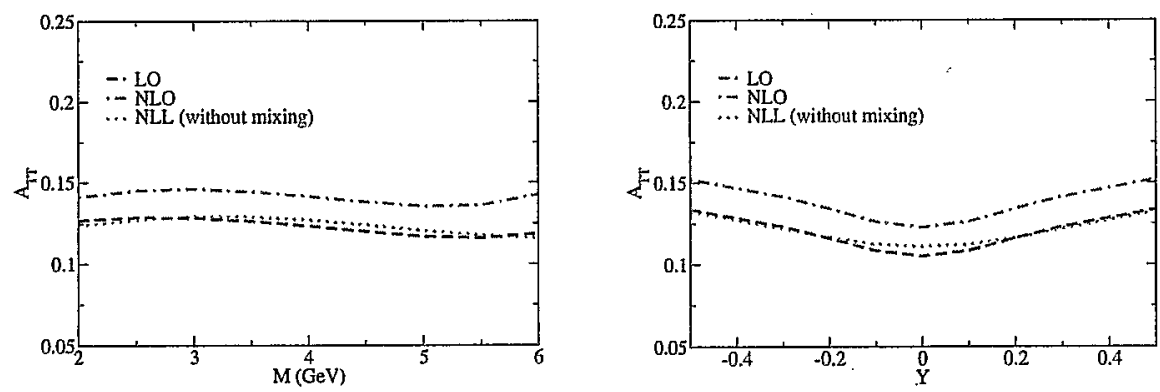

FIGURE 2. $A_{T T}$ for the J-PARC $p p$ collision at $\sqrt{s}=10 \mathrm{GeV}$. Left: invariant-mass distribution, right: rapidity distribution at $M=5 \mathrm{GeV}$.

\section{ACKNOWLEDGMENTS}

We thank S. Forte, D. de Florian and A. Mukherjee for useful discussions. The work of H.Y. is supported in part by a Research Fellowship of the Japan Society for the Promotion of Science. W.V. is supported by the U.S. Department of Energy under contract number DE-AC02-98CH108866.

\section{REFERENCES}

1. V. Barone et al. [PAX Collaboration], arXiv:hep-ex/0505054;

M. Maggiora et al. [ASSIA Collaboration], arXiv:hep-ex/0504011.

2. D. Dutta et al., Letter of Intent for "Physics of High-Mass Dimuon Production at the 50-GeV Proton Synchrotron".

3. G. Sterman, Nucl. Phys. B 281, 310 (1987);

S. Catani and L. Trentadue, Nucl. Phys. B 327, 323 (1989); 353, 183 (1991).

4. H. Shimizu et al., Phys. Rev. D 71, 114007 (2005); Nucl. Phys. Proc. Suppl. 157, 197 (2006).

5. R. Hamberg et al., Nucl. Phys. B 359, 343 (1991); 644, 403(E) (2002); R. V. Harlander and W. B. Kilgore, Phys. Rev. Lett. 88, 201801 (2002).

6. W. Vogelsang, Phys. Rev. D 57, 1886 (1998).

7. A. Vogt, Phys. Lett. B 497, 228 (2001); S. Catani et al., JHEP 0307, 028 (2003);

S. Moch and A. Vogt, Phys. Lett: B 631, 48 (2005).

8. T. O. Eynck, E. Laenen and L. Magnea, JHEP 0306, 057 (2003).

9. S. Catani et al., Nucl. Phys. B 478, 273 (1996).

10. M. Kramer, E. Laenen and M. Spira, Nucl. Phys. B 511, 523 (1998);

S. Catani, D. de Florian and M. Grazzini, JHEP 0201, 015 (2002).

11. A. Kulesza, G. Sterman and W. Vogelsang, Phys. Rev. D 66, 014011 (2002); 69, 014012 (2004).

12. C. Anastasiou, L. J. Dixon, K. Melnikov and F. Petriello, Phys. Rev. Lett. 91, 182002 (2003); Phys. Rev. D 69, 094008 (2004).

13. O. Martin et al., Phys. Rev. D 60, 117502 (1999).

14. G. Sterman and W. Vogelsang, JHEP 0102, 016 (2001); A. Mukherjee and W. Vogelsang, Phys. Rev. D 73, 074005 (2006); W. Vogelsang and H. Yokoya, arXiv:hep-ph/0607043; V. Ravindran, J. Smith and W. L. van Neerven, arXiv:hep-ph/0608308; P. Bolzoni, arXiv:hep-ph/0609073.

15. J. Soffer, Phys. Rev. Lett. 74, 1292 (1995).

16. M. Glück, E. Reya and A. Vogt, Eur. Phys. J. C 5, 461 (1998).

17. M. Glück, E. Reya, M. Stratmann and W. Vogelsang, Phys. Rev. D 63, 094005 (2001). 